Qummar Zaman*, Senan Alraho, and Andreas König

\title{
Gaussian Process Regression Based Robust Optimization with Observer Uncertainty for Reconfigurable Self-x Sensory Electronics for Industry 4.0
}

\author{
Auf Gaußscher Prozessregression basierende robuste Optimierung mit Beobachterunsicherheit für \\ rekonfigurierbare Self-x-Sensorelektronik für Industrie 4.0
}

DOI 10.1515/teme-2021-0061

\begin{abstract}
This paper presents a robust optimization technique for the reconfigurable measurement of sensory electronics for industry 4.0 to obtain a robust solution even in the presence of observer uncertainty using a cost-effective performance measurement method. The extrinsic evaluation of the proposed methodology is performed on an indirect current-feedback instrumentation amplifier (CFIA), which is a fundamental part of sensory systems. To reduce the CFIA device performance evaluation set-up cost, a low-cost test stimulus is applied to the circuit under test, and the output response of the circuit is examined to correlate with the device's performance parameters. Due to the complexity of the smart sensory electronics search space, the meta-heuristic optimization algorithm is being selected as an optimizer. For objective space or observer uncertainty, the Gaussian process regression from the Bayesian statistical regression process is used to estimate the uncertainty level efficiently. Six different classical metrics have been used to evaluate the regression model accuracy. The highest achieved average expected error metrics value is 0.313 , and the minimum value of correlation performance metrics is 0.908 . The device is implemented using $0.35 \mu \mathrm{m}$ austriamicrosystems technology.
\end{abstract}

Keywords: Measurement Uncertainty, Bayesian Statistical Regression, Gaussian Process Regression, Self-x Properties, Robust Optimization, Meta-heuristic Optimization.

Zusammenfassung: In diesem Beitrag wird ein robustes Optimierungsverfahren für die rekonfigurierbare Messung

*Corresponding author: Qummar Zaman, Lehrstuhl Kognitive Integrierte Sensorsysteme (KIS), TU Kaiserslautern Deutschland; and University of Engineering \& Techonolgy Taxila, Pakistan. E-Mail: qummar@eit.uni-kl.de.

Senan Alraho, Andreas König, Lehrstuhl Kognitive Integrierte Sensorsysteme (KIS), TU Kaiserslautern Deutschland. von sensorischer Elektronik für Industrie 4.0 vorgestellt, um eine robuste Lösung auch bei Unsicherheit des Beobachters mit einer kostengünstigen Leistungs- bzw. Tauglichkeitsmessmethode. Die extrinsische Bewertung der vorgeschlagenen Methodik wird an einem indirekten stromrückgekoppelten Instrumentenverstärker (CFIA) durchgeführt, der ein grundlegender Bestandteil von sensorischen Systemen ist. Um die Einrichtungskosten für die Bewertung der CFIA-Geräteleistung zu reduzieren, wird ein kostengünstiger Teststimulus auf den zu prüfenden Schaltkreis angewendet und die Ausgangsreaktion des Schaltkreises wird untersucht, um mit den Leistungsparametern des Geräts zu korrelieren. Aufgrund der Komplexität des Suchraums der intelligenten sensorischen Elektronik wird der meta-heuristische Optimierungsalgorithmus als Optimierer gewählt. Für den Zielraum oder die Beobachterunsicherheit wird die Gaußsche Prozessregression aus dem Bayes'schen statistischen Regressionsverfahren verwendet, um das Unsicherheitsniveau effizient zu schätzen. Es wurden sechs verschiedene klassische Metriken verwendet, um die Genauigkeit des Regressionsmodells zu bewerten. Der höchste erreichte durchschnittliche erwartete Fehler liegt bei 0,313, während die minimale Größe der PerformanceKorrelationsmetriken 0,908 beträgt. Der Baustein ist in 0,35 $\mathrm{mm}$ austriamicrosystems technology realisiert.

Schlüsselwörter: Messunsicherheit, Bayessche statistische Regression, Gaußsche Prozessregression, Self-x Eigenschaften, Robuste Optimierung, Meta-heuristische Optimierung.

\section{Introduction}

Artificial intelligence (AI) and machine learning (ML) are considered the equivalent of electricity for the twentieth century. The combination of AI and ML with rapidly grow- 
ing technologies, such as cloud computing, cyber-physical systems, and the industrial internet of things (I(I)oTs), accelerates the most revolutionary change in the industrial field known as industry 4.0 [1, 4, 7]. I(I)oTs and industry 4.0 devices highly rely on sensors and sensory electronics to measure data accuracy, flexibility, robustness, and long-term reliability [11]. Nevertheless, the performance of the smart sensory electronics system (SSES) declines gradually. These SSES have the self-x (self-optimize, selfcalibration, self-healing, etc.) properties with the fusion of $\mathrm{AI}$ and ML $[8,23]$. However, in a noisy environment, the system performance is unacceptable even after the calibration or optimization process [6]. Generally, there are three types of uncertainties that SSES might experience. Firstly, input uncertainty is defined by the perturbation in the design or search space variables mainly because of the fabrication or manufacturer errors. Variations due to the environment or operating conditions are classified as the second type of uncertainty. Lastly, observer or measurement uncertainty is mainly because of an imperfect observer (sensor or ADC). More details about different types of uncertainties for the SSES can be found in our previous research work [23].

Usually, the calibration methods include designing SSES with controllable tuning knobs plus performance evaluation set-up. As a consequence of a limited area and power constraints imposed by SSES, there is also ongoing research activity in our research group to introduce the optimum number of reconfigurable knobs to the analog front end without sacrificing its degrees of freedom notably [2]. Therefore, the main objective of this work is to figure out the optimization process using the low-cost indirect performance measurement method in the presence of observer uncertainty.

As far as system performance evaluation set-up is concerned, it can be classified into two different categories. The first category is on-chip direct performance measurement methods $[12,13]$. These methods are generally precise but consumed more area and power resources. In contrast, the second category is indirect measurement methods in which multiple performance parameters of the device under test are statistically evaluated by using simple test stimuli $[3,9,15,20,21]$. For example, a multitone signal is applied to the circuit under test in [21], and the output transient response of the system is being analyzed for indirect estimation of performance parameters. To further improve the estimation accuracy, two defect filters based alternative test flow concept is demonstrated in [20]. An analogous methodology is presented in [15], where the performance characteristics of ICs are predicted by using an envelope detector. However, for the application of SSES, the category of indirect measurements needed to be optimized due to limited computational resources. This research work presented the cost-effective total harmonics distortion (THD) based indirect measurement method to reduce the performance measurement set-up cost.

For handling the observer uncertainty, the concept of robust optimization (RO) has the capability to address such a scenario [18]. A well-known Bayesian statistical regression process, specifically the Gaussian process regression (GPR) that lies under the classification of probabilistic RO approach [25], is being selected for tackling the imperfect observer problem. GPR has been widely used in the fields of uncertainty quantification and design optimization. Besides a wide range of functional model fitting, it also provides the confidence interval for the prediction values $[19,25]$. The uncertainty usually is well represented by GPR, and these GPR models could consequently deliver a better estimation of uncertainty [22] that provides RO.

Furthermore, one more critical aspect of data forecasting and interpolation using GPR can be utilized to reduce wireless transmission energy (WTE), which is often the primary cause of energy consumption for smart measurement sensory nodes. Numerous amounts of literature are available to save the WTE by data compression or interpolation [16]. As far as optimizer selection is concerned, the derivative-based optimization techniques cannot be used due to discontinuity in the objective space [17]. In comparison, population-based meta-heuristic optimization algorithms function extremely well even in the discontinuous objective space environment. Therefore, a population-based particle swarm optimization (PSO) is selected as a robust optimizer for this research work for the sake of its simple implementation and fast convergence speed [14]. Finally, a reconfigurable wide swing current-feedback instrumentation amplifier (CFIA), an integral part of sensory readout electronics, is used as a device under test (DUT) for the extrinsic evaluation of the proposed optimizer. The biasing current, capacitors, feedback resistors, and the transistor's width of CFIA are serving as a calibration or tuning knob (TK) for its online calibration.

\section{Proposed Methodology}

The block diagram of the proposed optimization procedure is shown in Figure 1. As already mentioned, CFIA is used as a DUT and PSO as an optimizer for this experiment. The GPR block analyzes the output response of the DUT 


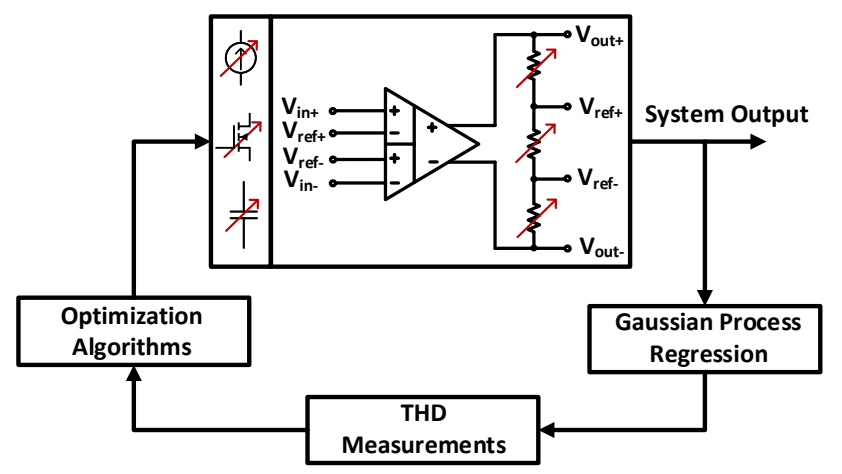

Fig. 1: Block diagram of proposed robust optimization.

for the prediction of the uncertainty level. After that, the THD-based low-cost indirect performance measurement block is being applied. It relies on the principle that design imperfection like limited gain bandwidth product (GBW), input common-mode range (ICMR), slew rate (SR), SNR, full-power-bandwidth, and the effective number of bits (ENBs) can be translated to non-linear distortion at the output of the closed-loop amplifier.

The flowchart of the proposed THD-based low-cost robust particle swarm optimizer (LCRPSO) is presented in Figure 2. It begins with random initialization of the position and velocity of the particles. In the next step, the sinusoidal signal is applied to the DUT, and uncertainty estimation is performed by using the GPR block. After the uncertainty estimation stage, the THD measurement is carried out. If the value of THD is above the specified threshold level, then the step signal is being applied to the system only in case of discovering a better fitness value as compared to the previous global best value. This adaptive application of step response saves many computation resources compared to performing the step response of DUT for every individual particle. Unfortunately, amplifier stability cannot be predicted from spectrum information obtained by the sinusoidal response. Therefore, the stability of the DUT is analyzed with the help of system step response. In the case of obtaining the particle's better fitness or cost function than the particle's global or personal best, the respective variable is being updated. The parameters of the LCRPSO are as follows:

Cognitive scaling factor $c_{1}=2$.

- Social scaling factor $\mathrm{c}_{2}=2$.

- Inertia weight $\mathrm{w}=\mathrm{w}_{\max }-\mathrm{k}\left(\frac{\mathrm{w}_{\max }-\mathrm{w}_{\min }}{\mathrm{k}_{\max }}\right)$ where $\mathrm{k}$ is current iteration, $\mathrm{k}_{\max }$ is maximum number of iterations, $\mathrm{w}_{\max }=0.9$, and $\mathrm{w}_{\min }=0.1$.

- Particle's initial velocity $=0$.

- Maximum velocity $=20 \%$ of upper limit of particle position.

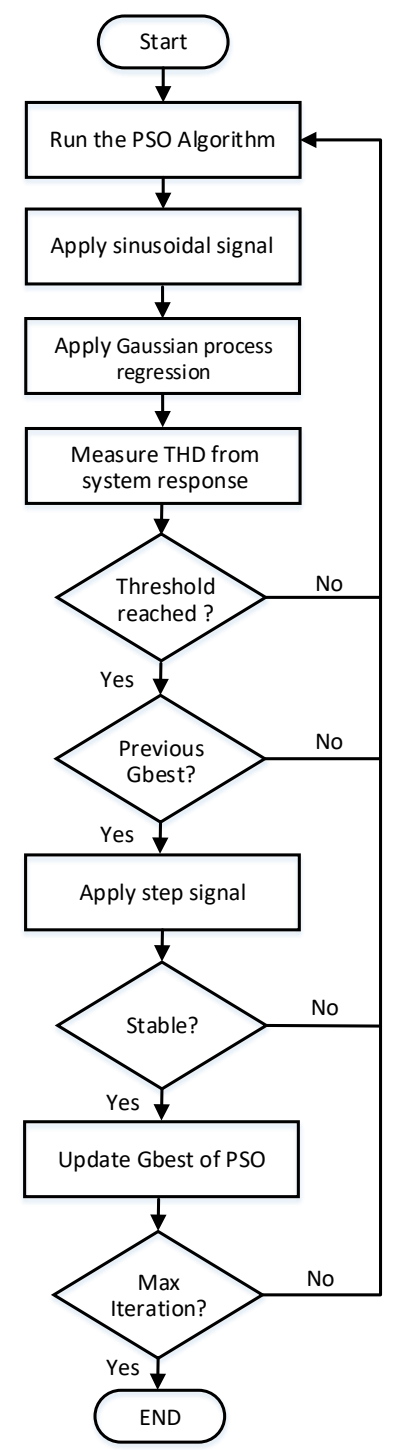

Fig. 2: Flowchart of the proposed THD-based low-cost robust particle swarm optimizer (LCRPSO).

Gaussian process regression is a constrained regression process to develop surrogate models [25]. Generally, the GPR model can be expressed as

$$
f(x) \sim G P\left(m(x), \kappa\left(x, x^{\prime}\right)\right)
$$

where $\mathrm{m}(\mathrm{x})$ represents the mean function and $\kappa\left(\mathrm{x}, \mathrm{x}^{\prime}\right)$ denotes the kernel function. The GPR is optimized by adapting the mean and kernel according to the training data set. We used the combination of the white kernel (to capture the noise variations) and exponential sine squared (to account for the periodic component) kernel for this experiment. 


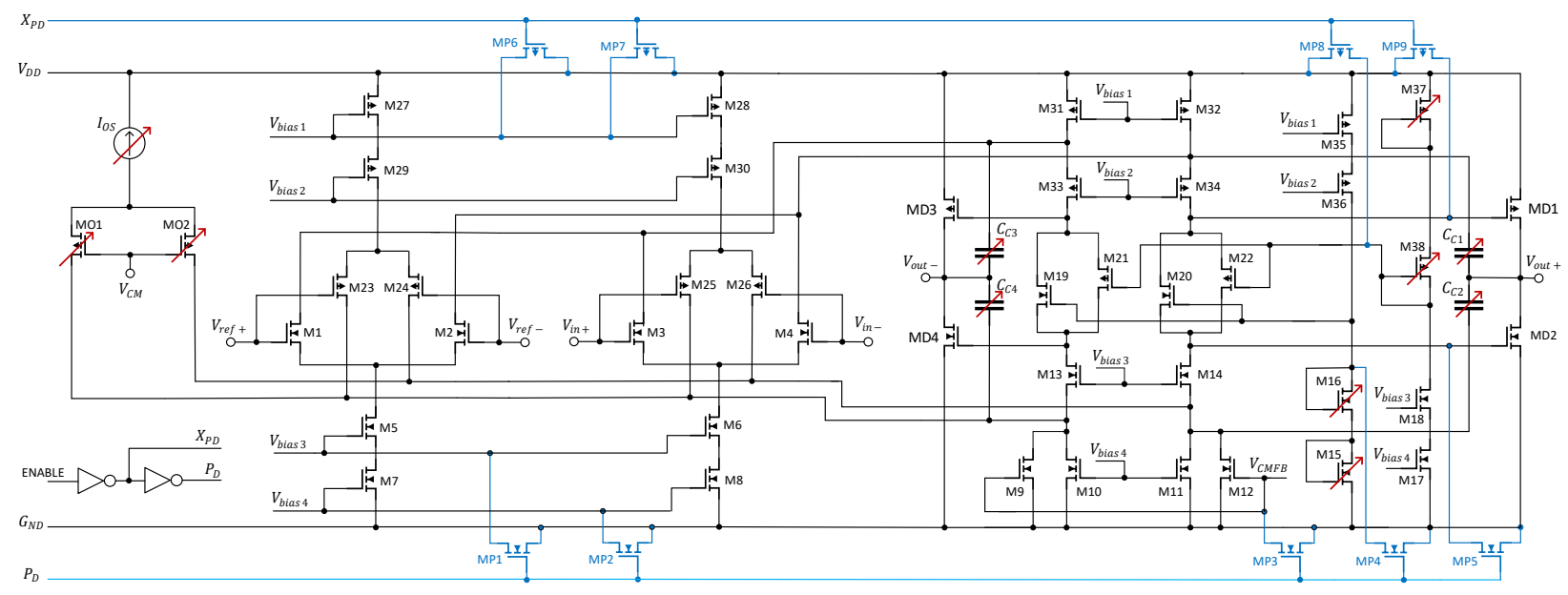

Fig. 3: Transistor level schematic diagram of the fully-differential reconfigurable CFIA.

Table 1: Calibration or tuning knobs of CFIA.

\begin{tabular}{llc}
\hline Reconfigurable element & Objective & No. of bits \\
\hline Feedback resistors & Gain adjustment & 8 \\
Compensation capacitors & SR, GBW, and Phase margin $(\mathrm{PM})$ & 4 \\
Class AB biasing circuit & DC power dissipation, first non-dominant pole & 30 \\
Biasing current & Major circuit characteristics, DC power consumption & 5 \\
Offset transconductance $\left(\mathrm{G}_{\mathrm{mso}}\right)$ & Offset voltage calibration $\left(\mathrm{V}_{\mathrm{os}}\right)$ & 31 \\
Current mirror biasing diodes & Saturation region & 22 \\
\hline
\end{tabular}

\section{Results and Discussion}

The extrinsic evaluation of the LCRPSO is performed on the complex objective space of reconfigurable CFIA. The CFIA is implemented using CMOS $0.35 \mu \mathrm{m}$ austriamicrosystems (ams) technology, and a transistor-level schematic diagram of the CFIA is shown in Figure 2. For this experiment, a $100 \mathrm{kHz}$ sinusoidal waveform with an amplitude of $2 \mathrm{~V}_{\mathrm{p}-\mathrm{p}}$ is used as a test stimulus, where the desired THD threshold is set to $-75 \mathrm{~dB}$. Similarly, for the step response, the square wave is applied with an amplitude of $2 \mathrm{~V}_{\mathrm{p}-\mathrm{p}}$ and a time period of 1 us. To minimize the channel length modulation effect, lengths of all transistors are kept constant to $1 \mu \mathrm{m}$, and the LCRPSO only adjusts the width of sensitive elements as given in Table 1. The LCRPSO and GPR blocks are implemented using python language, while the circuit is simulated using OCeaN (OCN) language in Cadence design tools.

The attained performance characteristics of the reconfigurable CFIA after performing the optimization process are presented in Table 2. As illustrated in this table, the proposed indirect measurement method using THD and pulse response revealed the successful optimization of the CFIA and fulfilled its performance characteristics. Fur-
Table 2: Performance characteristics of the CFIA after the optimization, $\mathrm{V}_{\mathrm{DD}}=3.3 \mathrm{~V}, \mathrm{~V}_{\mathrm{CM}}=1.65 \mathrm{~V}$.

\begin{tabular}{lc}
\hline CFIA design parameter & Value \\
\hline Differential DC gain (AVD) & $95.12 \mathrm{~dB}$ \\
Gain bandwidth product (GBW) & $78.43 \mathrm{MHz}$ \\
Phase margin $(\mathrm{PM})$ & $66.74^{\circ}$ \\
Slew rate (SR) & $207.61 \mathrm{~V} / \mu \mathrm{s}$ \\
Differential input linear range (VID) & $2.22 \mathrm{~V} \mathrm{P}-\mathrm{P}$ \\
Total harmonic distortion (THD) & $-85.88 \mathrm{~dB}$ \\
\hline
\end{tabular}

thermore, to avoid any lucky shot or randomness, this experiment is repeated over 15 independent simulations, and the statistical results of the optimization process are graphically demonstrated in Figure 4 by using the error bar graphs. Figure 5 shows the comparison between the noisy output signal and the predicted output signal using the proposed GPR-based robust optimizer with $95 \%$ confident intervals. The following six different error metrics are being exercised to evaluate the performance of the GPR: - Root mean square error is one of the most widely used performance metrics for the regression problems. It illustrates the average expected error (AEE) given as 

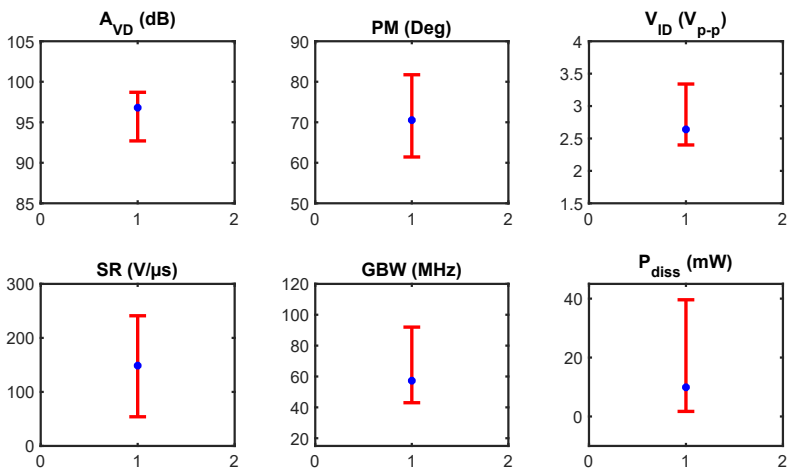

Fig. 4: Error bar diagram of the six different performance characteristics of the reconfigurable CFIA by using LCRPSO over 15 independent simulation runs.

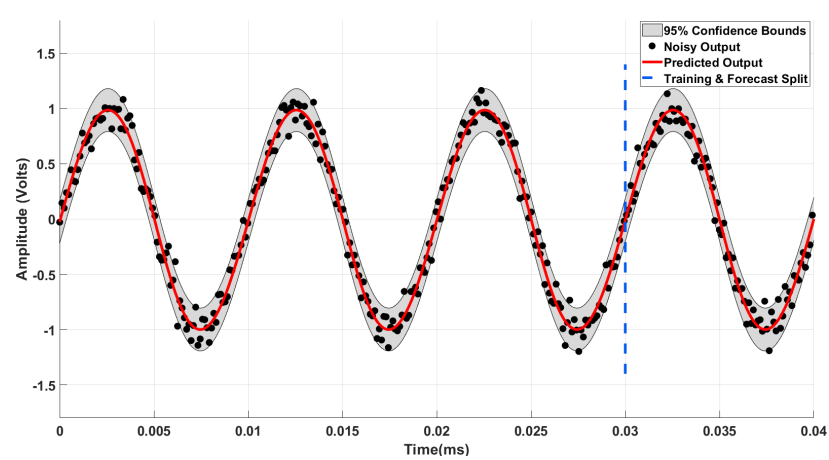

Fig. 5: The predicted output with the $95 \%$ confidence interval and illustration of data forecasting capabilities by the application of GPR block.

$$
R M S E=\sqrt{\frac{\sum_{\mathrm{i}=1}^{N}\left(y_{i}-\hat{y}_{i}\right)^{2}}{N}}
$$

where $\mathrm{y}_{\mathrm{i}}$ is the ground truth, $\hat{\mathrm{y}}_{\mathrm{i}}$ represents the estimated value and $\mathrm{N}$ is length of samples.

Pearson's correlation coefficient demonstrates the similarity among the true and estimated values express as

$$
p=100 \cdot \frac{\operatorname{cov}(y-\hat{y})}{\sigma_{y} \sigma_{\hat{y}}}
$$

where $\sigma_{y}$ and $\sigma_{\widehat{y}}$ are the standard deviations of true and predicted values.

- Mean absolute error characterizes the absolute difference between the predicted and true values defined as

$$
M A E=\frac{1}{N} \sum_{\mathrm{i}}^{N}\left|y_{i}-\hat{y}_{i}\right|
$$

- R-squared error also known as coefficient of determination determines the closeness of the data set to the
Table 3: Performance evaluation of the GPR by using six various error metrics.

\begin{tabular}{lll}
\hline Nr. & Error metrics & Value \\
\hline 1 & Root mean square error & 0.286 \\
2 & Pearson's correlation & 93.67 \\
3 & Mean absolute error & 0.313 \\
4 & R-squared error & 0.927 \\
5 & Adjusted-R squared error & 0.908 \\
6 & Figure of merit & 0.073 \\
\hline
\end{tabular}

predicted regression model defined as

$$
R^{2}=\frac{\sum_{\mathrm{i}}^{N}\left|y_{i}-\hat{y}_{i}\right|}{\sum_{\mathrm{i}}^{N}\left|y_{i}-\bar{y}\right|}
$$

where $\bar{y}$ is mean value of the data.

Adjusted-R squared is a variation of r-squared, that adjusts for the number of predictors in the model $(p)$ to the number of observation points $(N)$

$$
\bar{R}^{2}=1-\left(1-R^{2}\right) \frac{N-1}{N-p-1}
$$

Finally a Figure of merit presented in [5] equates the expected average error in the context of the data variation defined as

$$
F_{O} M=\frac{R M S E}{\sigma_{y}}
$$

The prediction efficiency of the GPR is listed in Table 3. MAE, FoM, and RMSE represent the AEE; therefore lower value corresponds to higher regressor accuracy. By contrast, $\mathrm{R}^{2}$, $\mathrm{p}$ and $\overline{\mathrm{R}}^{2}$ imply the correlation among the predicted and ground-truth value, hence the value closer to unity (100 for the $\mathrm{p}$ ) is assumed as the better predictor performance. It can be seen from Table 3 that the maximum value of the AEE metrics is 0.313 . For the correlation error metrics, the minimum magnitude is 0.908 , reflecting a nice correlation between the ground truth and estimation.

\section{Conclusions}

This work presented the low-cost extrinsic robust optimization of reconfigurable SSES by meta-heuristic optimization algorithms in the presence of observer uncertainty. The THD-based indirect measurement method is used to reduce the performance evaluation set-up cost. The potential of the proposed design methodology is illustrated by the successful optimization of performance characteristics of 
the reconfigurable CFIA. For tackling objective space uncertainty or observer uncertainty, the concept of GRP is being used. Besides uncertainly level estimation, the GPR can also help in forecasting the data to significantly reduce the WTE for wireless sensor network applications, as shown in Figure 5. As far as the intrinsic evaluation of the proposed methodology is concerned, our institute is also working on the extension and development of the universal sensor interface with self-x properties (USIX) chip [10] to provide a hardware platform for SSES [2]. Different pyramids of indirect measurement methods (intrusive, non-intrusive [24]) will be evaluated on this USIX 4.0 chip to explore the capabilities of these various indirect characteristics measurements methods for sensory electronics of industry 4.0. We are also working to extend low-cost performance measurement methodology to more sophisticated integrated circuits such as low pass filter for its optimization and tuning for future work [9].

\section{References}

[1] H. Abd and A. König. A compact four transistor cmos-design of a floating memristor for adaptive spiking neural networks and corresponding self- $x$ sensor electronics to industry 4.0. tm-Technisches Messen, 87(s1):s91-s96, 2020.

[2] S. Alraho, Q. Zaman, and A. König. Reconfigurable wide input range, fully-differential indirect current-feedback instrumentation amplifier with digital offset calibration for self-x measurement systems. tm-Technisches Messen, 87(s1): s85-s90, 2020.

[3] M. Andraud, H.-G. Stratigopoulos, and E. Simeu. Oneshot non-intrusive calibration against process variations for analog/rf circuits. IEEE Transactions on Circuits and Systems I: Regular Papers, 63(11):2022-2035, 2016.

[4] A. Angelopoulos, E. T. Michailidis, N. Nomikos, P. Trakadas, A. Hatziefremidis, S. Voliotis, and T. Zahariadis. Tackling faults in the industry 4.0 era-a survey of machine-learning solutions and key aspects. Sensors, 2020.

[5] M. J. Barragán, R. Fiorelli, G. Leger, A. Rueda, and J. L. Huertas. Alternate test of Inas through ensemble learning of on-chip digital envelope signatures. Journal of Electronic Testing, 27(3):277-288, 2011.

[6] M. J. Barragan, G. Leger, A. Gines, E. Peralias, and A. Rueda. On the limits of machine learning-based test: a calibrated mixed-signal system case study. In Design, Automation \& Test in Europe Conference \& Exhibition (DATE), 2017, pages 79-84. IEEE, 2017.

[7] R. Csalódi, Z. Süle, S. Jaskó, T. Holczinger, and J. Abonyi. Industry 4.0-driven development of optimization algorithms: A systematic overview. Complexity, 2021, 2021.

[8] F. Delaine, B. Lebental, and H. Rivano. In situ calibration algorithms for environmental sensor networks: A review. IEEE Sensors Journal, 19(15):5968-5978, 2019.
[9] E. A. Dri, G. M. Peretti, and E. A. Romero. A low-cost test strategy based on transient response method for embedded reconfigurable filters. International Journal of Electronics, pages 1-20, 2020.

[10] R. Freier. Ein universelles und dynamisch rekonfigurierbares interface fur eingebettete und intelligente multi-sensorsysteme mit self-x eigenschaften. 2015.

[11] A. Koenig. Integrated sensor electronics with self- $x$ capabilities for advanced sensory systems as a baseline for industry 4.0. In Sensors and Measuring Systems; 19th ITG/GMASymposium, pages 1-4. VDE, 2018.

[12] S. Lee. In Situ Automatic Analog Circuit Calibration and Optimization. PhD thesis, 2020.

[13] S. Lee, C. Shi, J. Wang, A. Sanabria, H. Osman, J. Hu, and E. Sánchez-Sinencio. A built-in self-test and in situ analog circuit optimization platform. IEEE Transactions on Circuits and Systems I: Regular Papers, 65(10):3445-3458, 2018.

[14] W. Liu, Z. Wang, X. Liu, N. Zeng, and D. Bell. A novel particle swarm optimization approach for patient clustering from emergency departments. IEEE Transactions on Evolutionary Computation, 23(4):632-644, 2018.

[15] V. Natarajan, S. Sen, A. Banerjee, A. Chatterjee, G. Srinivasan, and F. Taenzler. Analog signature-driven postmanufacture multidimensional tuning of $\mathrm{rf}$ systems. IEEE Design \& Test of Computers, 27(6):6-17, 2010.

[16] S. Pushpalatha and K. Shivaprakasha. Energy-efficient communication using data aggregation and data compression techniques in wireless sensor networks: A survey. In Advances in Communication, Signal Processing, VLSI, and Embedded Systems, pages 161-179. Springer, 2020.

[17] P. Rakshit, A. Konar, S. Das, L. C. Jain, and A. K. Nagar. Uncertainty management in differential evolution induced multiobjective optimization in presence of measurement noise. IEEE Transactions on Systems, Man, and Cybernetics: Systems, 44(7):922-937, 2013.

[18] P. Rakshit, A. Konar, and S. Das. Noisy evolutionary optimization algorithms-a comprehensive survey. Swarm and Evolutionary Computation, 33:18-45, 2017.

[19] C. E. Rasmussen. Gaussian processes in machine learning. In Summer school on machine learning. Springer, 2003.

[20] H.-G. Stratigopoulos and S. Mir. Adaptive alternate analog test. IEEE Design \& Test of Computers, 29(4):71-79, 2012.

[21] P. N. Variyam, S. Cherubal, and A. Chatterjee. Prediction of analog performance parameters using fast transient testing. IEEE Transactions on Computer-Aided Design of Integrated Circuits and Systems, 21(3):349-361, 2002.

[22] Y. Yang, S. Li, W. Li, and M. Qu. Power load probability density forecasting using gaussian process quantile regression. Applied Energy, 213:499-509, 2018.

[23] Q. Zaman and A. König. Self-x integrated sensor circuits immune to measurement noise in the presence of input perturbation by using robust optimization. tm-Technisches Messen, 86(s1):107-111, 2019.

[24] Q. Zaman, S. Alraho, and A. König. Low-cost indirect measurement methods for self-x integrated sensory electronics for industry 4.0. tm-Technisches Messen, 87(s1), 2020.

[25] Q. Zhou, P. Jiang, X. Huang, F. Zhang, and T. Zhou. A multi-objective robust optimization approach based on gaussian process model. Structural and Multidisciplinary Optimization, 57(1):213-233, 2018. 